\title{
Puentes extradosados: evolución y tendencias actuales
}

\section{Extradosed bridges: evolution and current trends}

\section{José Miguel Benjumea Royero}

Ingeniero Civil, candidato a magíster en Ingeniería Civil. Investigador de la Universidad Industrial de Santander. Bucaramanga, Colombia.

Contacto: josemiguelbenjumea@gmail.com

\section{Gustavo Chio Сho}

Ingeniero Civil, doctor en Ingeniería de Caminos, Canales y Puertos. Docente de la Universidad Industrial de Santander. Bucaramanga, Colombia.

Contacto: gchioch@uis.edu.co

\section{Esperanza Maldonado Rondón}

Ingeniera Civil, doctora en Ingeniería de Caminos, Canales y Puertos. Investigadora de la Universidad Industrial de Santander. Bucaramanga, Colombia.

Contacto: emaldona@uis.edu.co

Fecha de recepción: 10 de noviembre de 2011

Fecha de aceptación: 17 de abril de 2012

Clasificación del artículo: Revisión

Palabras clave: comportamiento estructural, diseño de puentes, puentes extradosados.

Key words: structural behavior, design of bridges, extradosed bridges.

\section{RESUMEN}

Desde su aparición en el año 1994, los puentes extradosados han tomado un lugar importante para proyectos de carretera y ferrocarril, compitiendo en el rango de luces medias con los puentes atirantados y con los de viga cajón pretensada construidos por voladizos sucesivos. Regionalmente, estos puentes han sido implementados con mayor auge en Asia, pero los recientes proyectos desarrollados en América y Europa demuestran el interés que empieza a generar este tipo estructural. Consecuentemente, este trabajo tiene el propósito de proveer una revisión de la evolución de 
los puentes extradosados, desde su origen hasta su aplicación actual; además, se pretende evidenciar, a través de los diferentes puentes que han sido construidos, que están en construcción y que se encuentran en fase de proyecto, las tendencias estructurales adoptadas. Finalmente, y como conclusión de este trabajo, se realiza el análisis de las principales ventajas y desventajas de esta tipología, en comparación con los puentes atirantados y los de pretensado de viga cajón.

\section{ABSTRACT}

Since their appearance in 1994, extradosed bridges have played an important role in road and railway projects, competing (in medium span lengths) with cables-stayed and prestressed box girder bridges. At a regional level, extradosed bridges have been implemented more frequently in Asia, but recent projects developed in Europe and America show a growing interest in this typology. Consequently, this paper is aimed at offering a survey viewpoint on the evolution of extradosed bridges, from their origin to their current applications. This paper is also intended to show the adopted structural trends by presenting existing extradosed bridges, bridges under construction, and newly designed bridges. Finally, the main advantages and disadvantages of this type of bridges are analyzed and compared to cablestayed and prestressed box girder bridges.

\section{INTRODUCCIÓN}

Los puentes pretensados de viga cajón y los atirantados son dos tipologías que han sido adaptadas en múltiples proyectos para salvar luces medias y que, actualmente, hacen parte de las estructuras clásicas. En el año 1994, los japoneses construyeron el puente Odawara Blueway, inspirados en la propuesta realizada por Mathivat para el viaducto Arrét Darré [1]. Este puente, reconocido como extradosado, reúne algunas características de las otras dos tipologías, resultando económicamente competitivo con éstas. Las bondades ofrecidas por los puentes extradosados han sido foco de atención de investigadores e ingenieros proyectistas, lo cual ha permitido proponer criterios de diseños aplicables a este tipo estructural [2] y, de forma paralela, se ha logrado su evolución, incorporando nuevas tecnologías y motivando el desarrollo de materiales. Sin embargo, los puentes extradosados siguen siendo desconocidos en algunos países, en donde para proyectos de carretera y ferrocarril se opta por las tipologías convencionales. Por consiguiente, en este trabajo

se presenta una revisión de los puentes extradosados construidos, en construcción y en fase de proyecto, mostrando a su vez la evolución que han tenido desde el inicio de su implementación, su campo de aplicación y las tendencias actuales en su implementación. Finalmente, se presenta un análisis de las ventajas y desventajas de su aplicación.

\section{REALIZACIONES A NIVEL MUNDIAL}

El crecimiento de la popularidad de los puentes extradosados, como solución para proyectos de carretera, ha sido significativamente más acelerado en comparación al de otras nuevas tipologías como los puentes con atirantamiento inferior, y con atirantamiento combinado superior e inferior. Desde su introducción en el año 1994 con la construcción del puente Odawara Blueway en Japón, se han construido, hasta la fecha, 110 puentes, otros ocho se encuentran en construcción, y dos están en fase de proyecto. De estos, se puede concluir que la mayoría de las apariciones a nivel 


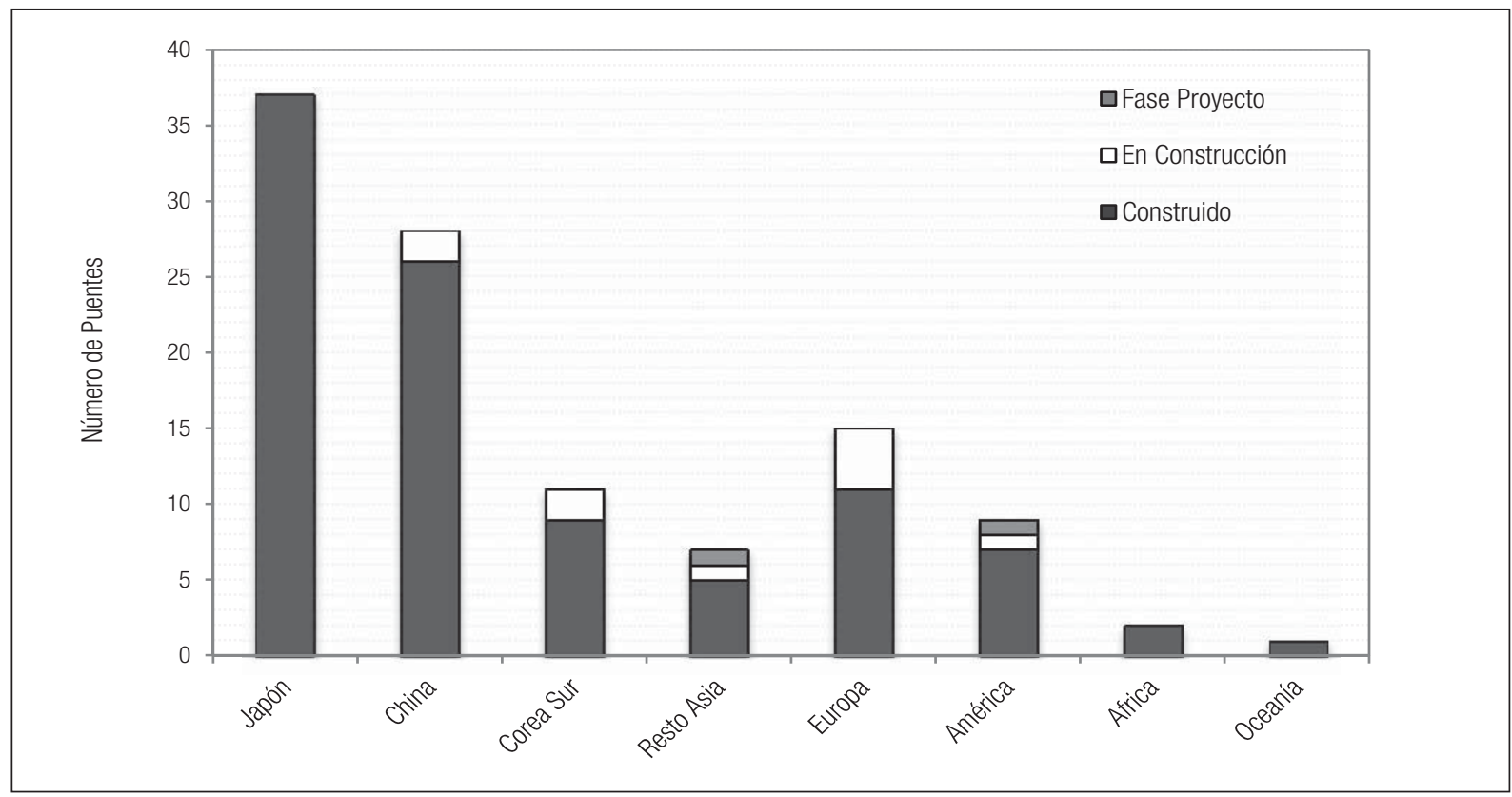

Figura 1. Relación del número de puentes extradosados construidos, en construcción y en fase de proyecto, de acuerdo a la ubicación regional.

Fuente: elaboración propia

mundial están localizadas en el continente asiático (83 del total), seguidos de Europa (15), América (9), África (2) y Oceanía (1), ver figura 1. En la tabla 1 se presenta un resumen de las principales características geométricas, las cuales se detallan en la figura 2, de algunos de los puentes estudiados, ordenados de menor a mayor longitud en el vano principal.

El éxito obtenido con la construcción de los primeros puentes extradosados en Japón, que es hasta la fecha el país con mayor número de ejemplares, se expandió rápidamente a los países aledaños. En China, el primer puente extradosado, puente Wuhu Yang-Tze, se construyó en el año 2000, y en Corea del Sur aparecen los puentes extradosados en el año 2002 con la construcción del puente Nocksan [26]. Desde entonces, los puentes extradosados se han convertido en una tipología bien recibida en el continente asiático, demostrado a partir de los 77 puentes construidos, cinco en construcción y uno en fase de proyecto. En Eu- ropa, la construcción de los puentes extradosados se ha visto limitada por la falta de las normativas para el diseño de los cables extradosados y las altas restricciones impuestas, como en el caso de Alemania, donde se impide el uso de tendones de preesfuerzo externo por fuera de la viga [5], aunque ya aparecen algunas normativas y guías de diseño, como [27] y [28], en la que se definen de manera más clara los criterios de diseño para cables extradosados, distinguiéndolos de los

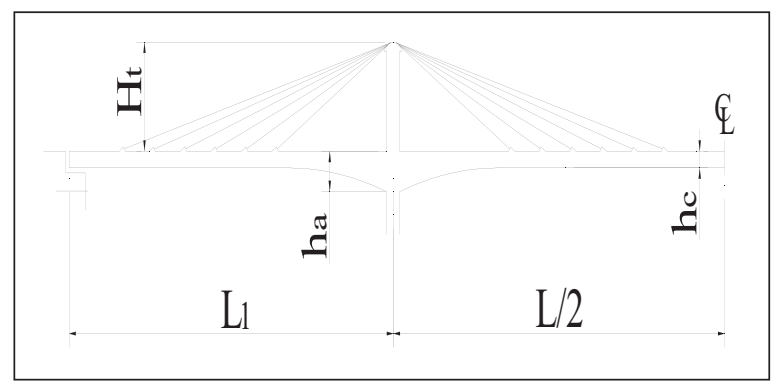

Figura 2. Nomenclatura de las dimensiones principales estudiadas.

Fuente: elaboración propia 
Tabla 1. Principales puentes extradosados.

\begin{tabular}{|c|c|c|c|c|c|c|c|c|}
\hline Nombre del Puente & País & Terminación & $\mathrm{L}(\mathrm{m})$ & $\mathrm{L} 1 / \mathrm{L}$ & $\mathrm{L} / \mathrm{Ht}$ & L/ha & $\mathrm{L} / \mathrm{hc}$ & Ref. \\
\hline Puente sobre el río Deba & España & 2003 & 66 & 0,64 & - & 24,4 & 24,4 & [3] \\
\hline Saint Rémy de Maurienne & Francia & 1996 & 80,8 & 0,60 & 13,69 & 36,7 & 36,7 & [4] \\
\hline Fukaura o Hukaura & Japón & 2002 & 90 & 0,69 & 10,59 & 30,0 & 36,0 & [5] \\
\hline $\begin{array}{l}\text { Tercer Puente sobre el Río } \\
\text { Acre }\end{array}$ & Brasil & 2006 & 90 & 0,60 & 7,50 & 36,0 & 45,0 & [6] \\
\hline Korong Extradosed & Hungría & 2004 & 91.392 & 0,57 & 9,67 & 36,6 & 36,6 & [7] \\
\hline Choqueyapu & Bolivia & 2010 & 92.5 & 0,57 & 5,85 & 26,4 & 44,0 & [8] \\
\hline Orkojahuira & Bolivia & 2010 & 103 & 0,64 & 6,52 & 29,4 & 49,0 & [8] \\
\hline Yashiro Sur & Japón & 1996 & 105 & 0,62 & 8,75 & 42,0 & 42,0 & [9] \\
\hline $\begin{array}{l}\text { Puente Integración Brasil- } \\
\text { Peru }\end{array}$ & Brasil & 2006 & 110 & 0,59 & 7,33 & 32,8 & 46,8 & [6] \\
\hline Second Vivekananda & India & 2007 & 110 & 0,50 & 7,86 & 31,4 & 31,4 & {$[10]$} \\
\hline Riga South & Letonia & 2008 & 110 & 0,70 & 8,59 & 45,8 & 45,8 & [11] \\
\hline Domivinski & Croacia & 2006 & 120 & 0,60 & 10,00 & 33,8 & 33,8 & {$[12]$} \\
\hline Santanigawa & Japón & 1999 & 121 & $0 ., 48$ & 12,8 & 18,6 & 48,3 & [13] \\
\hline Odawara Blueway & Japón & 1994 & 122 & 0,60 & 11,40 & 34,9 & 55,5 & [1] \\
\hline Kantutani & Bolivia & 2010 & 122 & 0,55 & 7,72 & 34,9 & 58,1 & [8] \\
\hline Shin-Meisei & Japón & 2004 & 122 & 0,67 & 7,41 & 34,9 & 34,9 & {$[14]$} \\
\hline Viaducto de Trois-Bassins & La Reunión (Fra) & 2008 & 126 & - & 6,63 & 29,0 & 51,0 & {$[15]$} \\
\hline Shin-Karato Oeste & Japón & 1998 & 140 & 0,53 & 11,48 & 40,0 & 56,0 & {$[16]$} \\
\hline Sunniberg & Suiza & 1998 & 140 & - & 10,14 & 127,0 & 127,0 & {$[12]$} \\
\hline New Pearl Harbor Memorial & USA & E.C. & 157 & 0,48 & 6,95 & 31,0 & 45,0 & {$[12]$} \\
\hline Tsukuhara & Japón & 1997 & 180 & 0,42 & 11,25 & 32,7 & 60,0 & {$[17]$} \\
\hline Kanisawa o Shouyou & Japón & 1998 & 180 & 0,55 & 8,14 & 32,1 & 54,5 & {$[18]$} \\
\hline Himi-Yume & Japón & 2004 & 180 & 0,51 & 9,09 & 45,0 & 45,0 & [5] \\
\hline Canada Line o North Arm & Canadá & 2008 & 180 & 0,77 & 8,18 & 52,9 & 52,9 & [19] \\
\hline Mactan Mandaue & Filipinas & 1999 & 185 & 0,60 & 10,16 & 37,8 & 59,7 & [20] \\
\hline Sannohe-Boukyo & Japón & 2005 & 200 & 0,50 & 8,00 & 30,8 & 57,1 & [5] \\
\hline Miyakodagawa & Japón & 2001 & 213 & 0,63 & 10,64 & 32,7 & 53,2 & {$[21]$} \\
\hline Tokunoyawa Hattoku & Japón & 2006 & 220 & 0,64 & 9,78 & 33,8 & 62,9 & [5] \\
\hline Hemaxi & China & 2006 & 230 & 0,54 & - & - & - & {$[22]$} \\
\hline Golden Ears & Canadá & 2009 & 242 & 0,50 & 6,05 & 53,8 & 89,6 & {$[12]$} \\
\hline Koror-Babeldaob & Rep. de Palaos & 2002 & 247 & 0,33 & 9,15 & 35,3 & 70,6 & {$[23]$} \\
\hline Ibi Gawa & Japón & 2001 & 271.5 & 0,58 & 9,05 & 37,2 & 63,1 & {$[24]$} \\
\hline Kiso Gawa & Japón & 2001 & 275 & 0,58 & 9,17 & 37,7 & 64,0 & [24] \\
\hline Wuhu Yang-Tze & China & 2000 & 312 & 0,58 & 9,38 & 20,8 & 20,8 & {$[25]$} \\
\hline
\end{tabular}

Fuente: elaboración propia

Notas: (E.C.) En construcción; (-) Sin dato; para los puentes de 2 vanos se ha hecho una analogía con los de tres vanos, asumiendo la luz principal como el $80 \%$ de la suma de la longitud de los dos vanos, con el fin de poder establecer las comparaciones paramétricas mostradas en las figuras 13 a 19. 


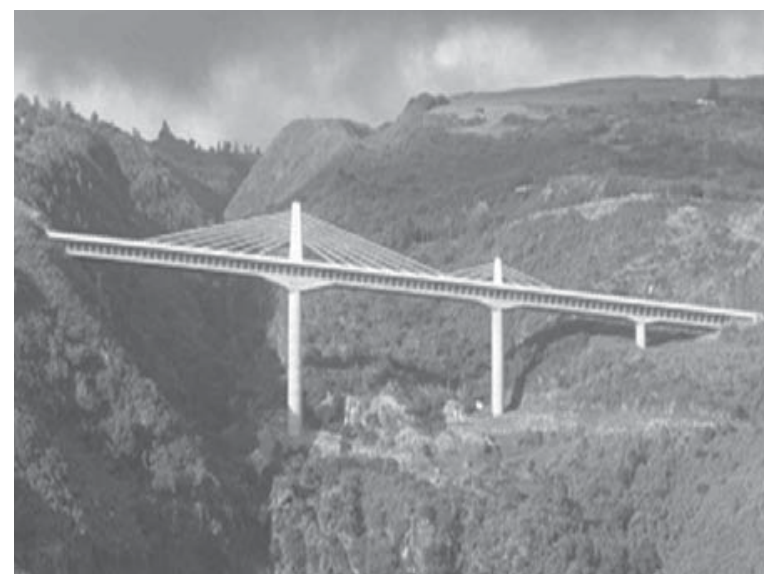

Figura 3. Viaducto de Trois Bassins en La isla Reunión.

Fuente: tomado de [29]

tirantes en puentes atirantados. El primer puente extradosado construido en Europa fue el Puente de Saint Rémy de Maurienne, terminado en el año 1996 en Francia [4]. Con la construcción en el año 2008 del Viaducto de Trois Bassin [15] en la Isla Reunión, perteneciente a Francia, ver figura 3, se completan en Europa once puentes construidos mientras que otros cuatro se encuentran en construcción.

La introducción de los puentes extradosados en el continente americano se dio en Brasil con la construcción del Tercer Puente sobre el Río Acre, y del Puente de La Integración Brasil-Perú, ambos en el año 2006. Los puentes North Arm y Golden Ears en Canadá, y los puentes Trillizos en Bolivia, ver figura 4, completan la lista de realizaciones en América. Actualmente, se ha iniciado la construcción del Puente Quinnipac en Estados Unidos [30] y se encuentra en fase de proyecto otro puente para cruzar el Río St. Croix en Minnesota [31]. En África, con ayuda de empresas japonesas, se han construido dos puentes extradosados: el Puente Blue Nile Gorge en Etiopía, terminado en el año 2008 [32] y el Rades-La Goulette en Túnez, finalizado en el año 2009 [33]. El

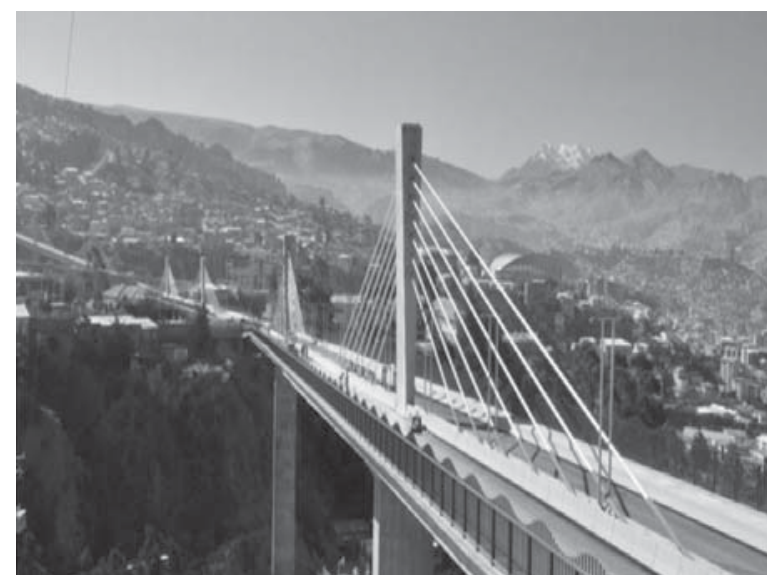

Figura 4. Puentes Los Trillizos en Bolivia. Fuente: tomado de [34]

único puente extradosado construido en Oceanía es el Puente Koror-Babeldaob en la República de Palaos, ver figura 5, diseñado y construido por empresas japonesas.

\section{EVOLUCIÓN}

Los primeros puentes extradosados construidos en Japón se caracterizaron por usar pilares conectados monolíticamente al tablero, y por utilizar

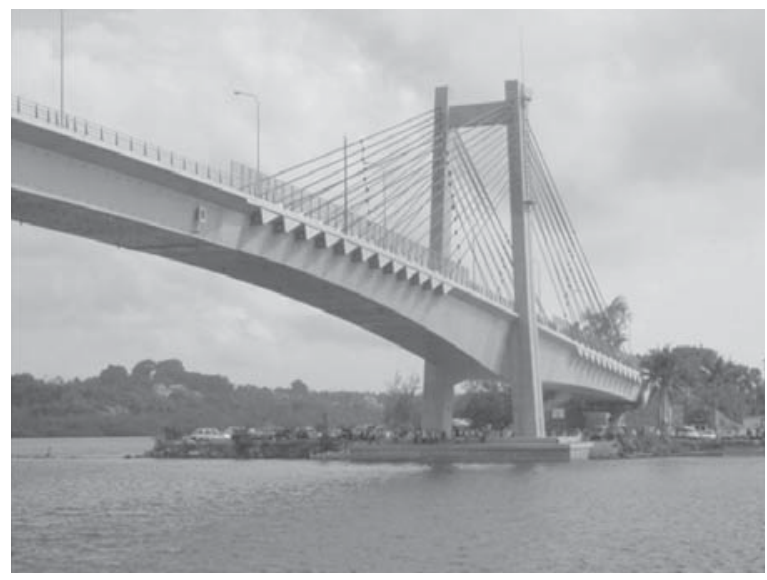

Figura 5. Puente Koror-Babeldaob en La República de Palaos.

Fuente: tomado de [12] 


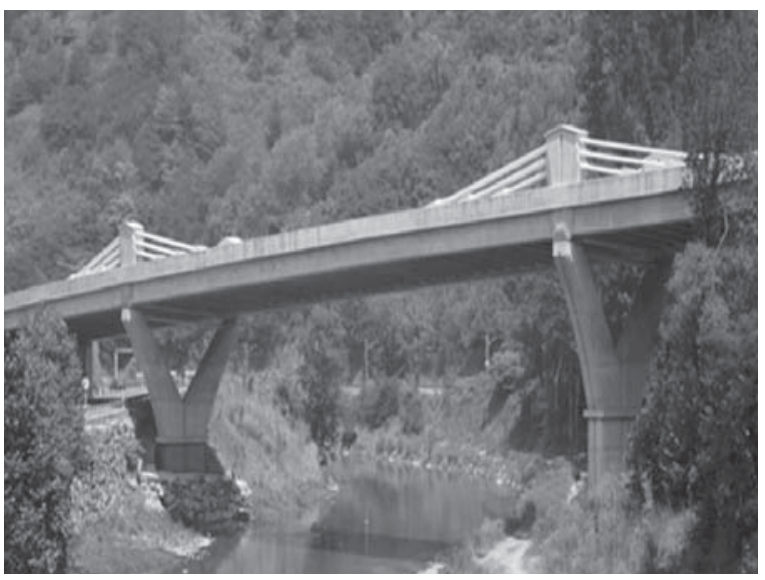

Figura 6. Puente Sobre el Río Deba en España.

Fuente: tomado de [35]

dos planos de cables anclados a los pilonos y a lo largo del borde del tablero. Desde el punto de vista estructural, esta configuración genera mayor rigidez transversal, reduciendo los efectos dinámicos en la estructura producto de la acción del viento y del sismo. Además, debido a que la oscilación de tensión en los cables, por efecto de la carga viva, no es alta, estos elementos podrían estar bajo esfuerzos máximos de . En el año 1996, con la construcción del Puente Saint Rémy de Maurienne, apareció una nueva configuración para los puentes extradosados, sin embargo, los planos de suspensión empleados hicieron poco atractiva esta solución estructural. El puente sobre el Río Deba en España, abierto al servicio en el año 2003, ver figura 6, también usó este arreglo.

La construcción, en el año 1995, del Puente de Sunniberg en Suiza, marcó un cambio en la concepción estructural de los puentes extradosados, ver figura 7, llegando a ser considerada como uno de los proyectos con mayor ingenio durante su concepción que han sido construidos hasta la fecha [36]. En esta estructura se empleó uno de los esquemas usados en puentes atirantados para proveer rigidez y estabilidad, mediante el uso de torres rígidas, lo que permitía tableros más es-

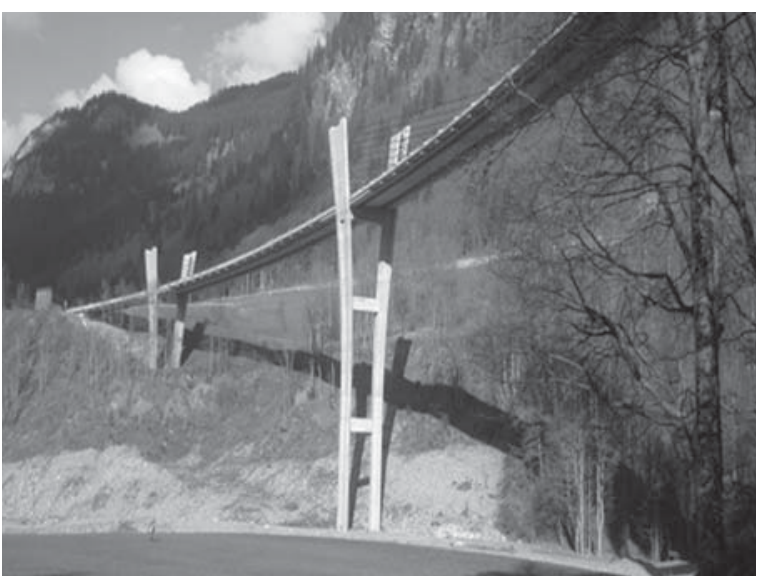

Figura 7. Puente Sunniberg en Suiza.

Fuente: tomado de [37]

beltos y daba una apariencia más ligera. En estos puentes, debido a que las oscilaciones de tensión en los tirantes son mayores que en los primeros puentes construidos en Japón, la fuerza de tesado en los cables tiene que ser reducida para evitar problemas por fatiga.

En los últimos diez años, la evolución de los puentes extradosados ha ido de la mano del uso de tableros híbridos, tal y como sucedió en los puentes atirantados. En el año 2000 se construyó el Puente Wuhu sobre el Río Yang-Tze en China, ver figura 8 , que es, hasta la fecha, el record mundial de longitud principal para los puentes extradosados. En esta estructura se empleó un tablero doble, separando el tráfico de automóviles y el de trenes, y se rigidizó el sistema lateral y verticalmente por medio de la acción híbrida entre la losa de concreto del tablero y una cercha metálica ubicada entre los dos niveles [25].

Buscando una reducción del peso de la superestructura y, al mismo tiempo, incrementar la luz abarcada, en los Puentes Ibi y Kiso Gawa construidos en el año 2002 en Japón, se empleó una estructura compuesta para los vanos centrales [38], ver figura 9. La superestructura consiste en un elemento híbrido, en donde los primeros 


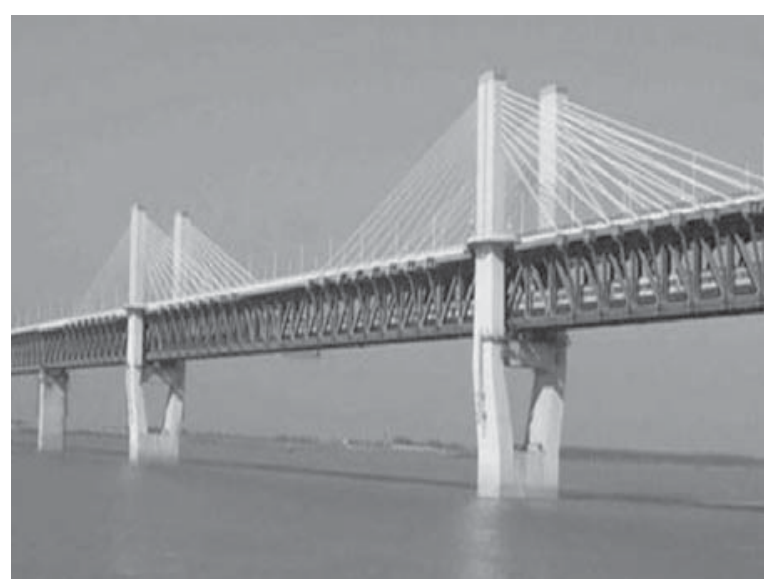

Figura 8. Puente Wuhu sobre el Río Yang-Tze en China.

Fuente: tomado de [39]

90 metros, en el vano principal, son elementos de concreto prefabricado soportados por cables, y en el vano central se dispone una viga metálica con losa ortotrópica en concreto, de $100 \mathrm{~m}$ de longitud, la cual es continua con las secciones de concreto gracias al uso de segmentos especiales de conexión prefabricados [24]. Esta solución también fue implementada en el Puente Koror Babeldaop.

En el Puente Himi Yume, construido en Japón, en el año 2004, se emplearon tableros con almas metálicas corrugadas, ver figura 10. Este concepto, introducido en el año 1986 con la construcción del puente Cognac en Francia [41], busca reducir el peso de la superestructura, aumentar la eficiencia de transmisión del pretensado a las losas superior e inferior del tablero y disminuir las labores de trabajo [42], [43]. Debido a que esta técnica no había sido implementada en puentes extradosados, se construyó un modelo parcial del puente a escala $1: 1 / 2$, buscando verificar el comportamiento de los miembros diagonales en los puntos de anclaje. Los ensayos realizados permitieron verificar la seguridad del puente y la validez de los diseños [44]. Esta técnica también fue implementada en el puente Rittoh en Japón, abierto al servicio en el año 2005.

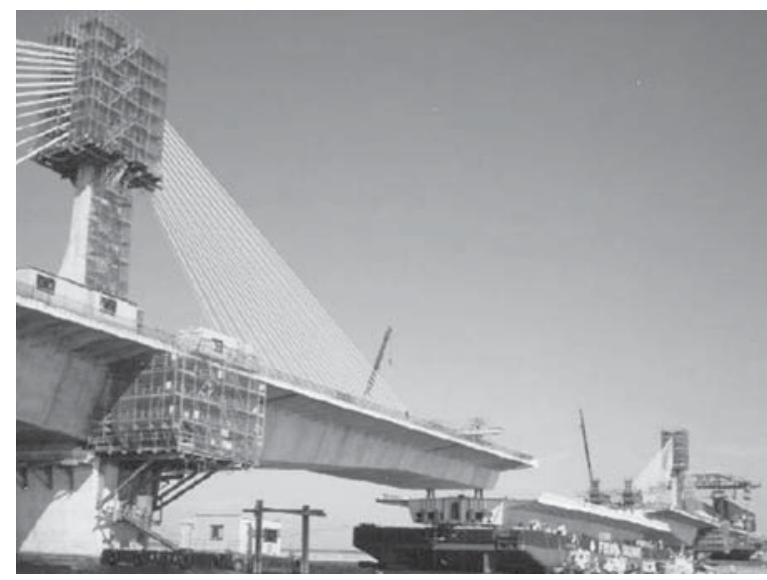

Figura 9. Erección de la viga metálica en el Puente Ibi Gawa en Japón.

Fuente: tomado de [40]

Recientemente se construyó en Canadá el Puente Golden Ears, el cual es el primer puente hibrido entre un atirantado y un extradosado, ver figura 11. Las restricciones impuestas por la cercanía a un aeropuerto, en relación a la altura de los pilonos, no permitían el uso de un puente atirantado o uno colgante. Entonces, se optó por un puente híbrido, en el cual "existe un poco de la conducta de puente extradosado particularmente cerca de las pilas... pero una gran parte de la carga es tomada por los tirantes" (Bergman, citado en [46]). El tablero del puente principal está compuesto

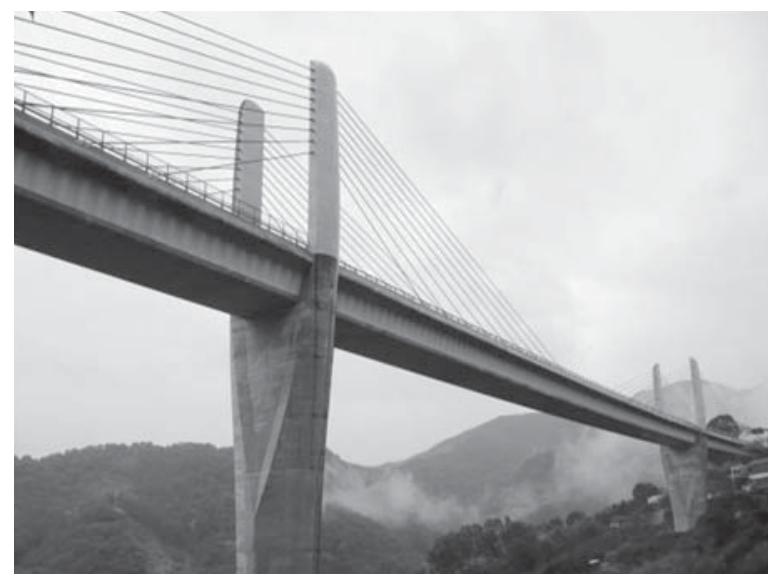

Figura 10. Puente Himi Yume en Japón.

Fuente: tomado de [45] 


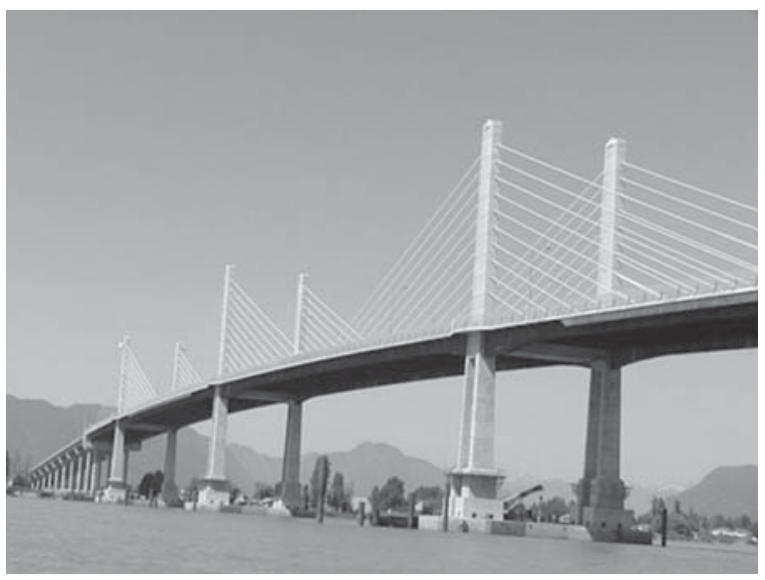

Figura 11. Puente Golden Ears en Canadá.

Fuente: tomado de [47]

por una losa en concreto, que forma una sección híbrida con dos vigas metálicas longitudinales ubicadas en los bordes y conectadas entre sí por vigas transversales en acero.

Aunque en su mayoría los puentes extradosados se construyen por el método de voladizos sucesivos, los avances tecnológicos en el área de la construcción fueron adaptados a esta tipología, lo que ha permitido construir puentes extradosados por el método de empuje, como es el caso del puente ferroviario de Sapporo en Japón, que fue el primer puente de este tipo y por lo tanto se requirieron consideraciones especiales durante su construcción [48]; y el puente Riga sobre el río Daugava en Letonia, abierto al tránsito en el año 2008, en donde, a diferencia del puente de Sapporo, el tablero está conformado por vigas metálicas y una losa en concreto [49], mostrando así la adaptabilidad de los puentes extradosados a diferentes procesos constructivos y materiales.

\section{CAMPO DE APLICACIÓN}

Se ha tratado de establecer el campo de aplicación en el cual los puentes extradosados son económicamente competitivos en comparación a otras tipologías, sin embargo, aún no hay un rango definido. De acuerdo a [50] los puentes extradosados son ideales para luces principales entre 100 y 150 metros, rango similar al propuesto por [51], en donde se establece el óptimo para luces principales entre 80 y 150 metros. Estudios desarrollados en [52], donde comparan puentes extradosados, atirantados, con atirantamiento combinado superior e inferior, y de hormigón pretensado construidos por voladizos sucesivos, demuestran que los primeros aventajaban económicamente a los otros tipos hasta luces principales de $225 \mathrm{~m}$. En [20] se concluye que para luces entre 150 y $200 \mathrm{~m}$, aunque los puentes extradosados resultan ser más económicos que los puentes pretensados de viga cajón, la diferencia de costos no es tan grande y podría afectarse por alteraciones en los precios locales de los materiales o por los efectos de las condiciones topográficas y geológicas del sitio. Teniendo en cuenta factores como la sección transversal de la viga, la sección y altura de pilonos, la luz del vano principal, y los precios unitarios de los cables, en [53] se concluye que, para luces medias, la solución de diseño óptima entre un puente atirantado y un puente extradosado, tiende hacia el último, sobre todo cuando los precios unitarios de los cables son bajos y las solicitaciones permanentes por vibración en los cables son bajas.

De los puentes extradosados estudiados en este trabajo, con datos de luz principal disponibles, se puede concluir que la mayoría de las aplicaciones se han usado para luces principales entre 100 y 150 metros (55 del total), seguido de aplicaciones con luces entre 50 y 100 metros (25), ver figura 12. El número de aplicaciones para luces principales mayores a 150 metros es de 21, aún sin sobrepasar al segundo rango; sin embargo, la evolución de estos puentes, a través del uso de tableros mixtos y metálicos, hace pensar que en poco tiempo el número de soluciones con luces mayores a 150 metros se incrementará. 


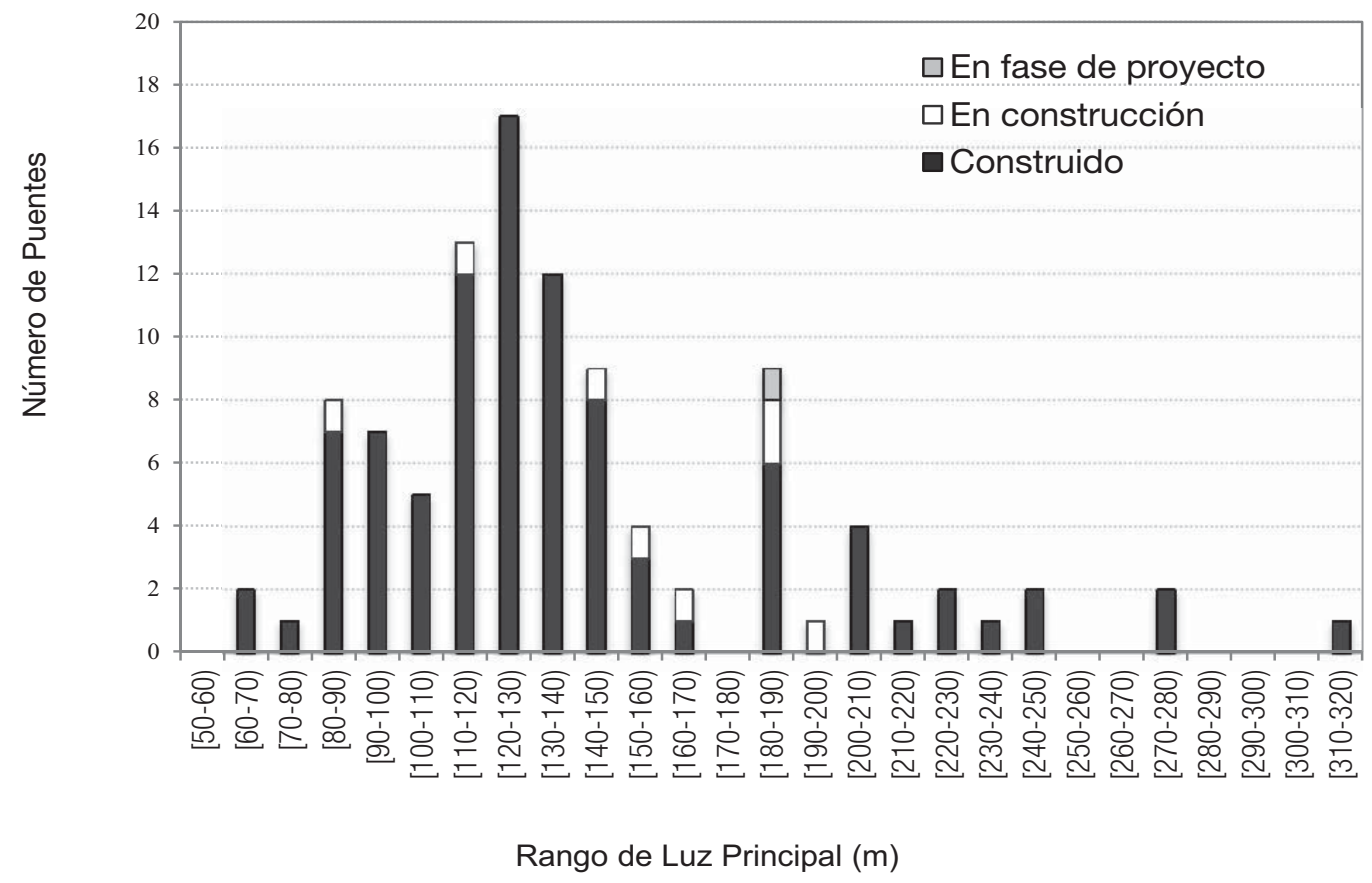

Figura 12. Relación de número de puentes construidos, en construcción y en proyecto, de acuerdo al rango de luz principal.

Fuente: elaboración propia

\section{TENDENCIAS ACTUALES}

Al realizar un análisis detallado de las características principales del puente (canto del tablero, altura de la torre, vinculación tablero-pilar y longitud del vano lateral), siguiendo lo presentado en [12], pero con una mayor base de datos, se pueden concluir las siguientes tendencias:

- En general se prefiere el uso de tableros con altura variable, con canto en la sección de apoyo en pila entre $\mathrm{L} / 30 \mathrm{y} \mathrm{L} / 35$, que concuerda con la recomendación de [54], y entre L/45 y L/50 para la sección en el centro de luz, empatando con la recomendación propuesta por [55], ver figura 13.

- A medida que se incrementa la luz principal del puente, se prefiere un acartelamiento con $\mathrm{ha} / \mathrm{hc}$ mayor a $1.5 \mathrm{y}$ tablero unido rígidamen- te a los pilares, siendo la excepción el puente Wuhu Yang-Tze, ver figuras 14 y 15. Relaciones ha/hc menores a 1.5 , son empleadas más para puentes con tablero apoyado en las pilas y luces principales menores a 150 metros.

- Al analizar el tipo de vinculación entre el tablero y los pilares, en función de la región en que se construyen, la cual está estrechamente relacionado con la amenaza sísmica, se observa una fuerte paridad para las dos vinculaciones típicas empleadas, ver figura 16.

- En general, se prefieren alturas de torres entre $\mathrm{L} / 12$ y L/8, que es el rango propuesto por [56]. El uso de torres con alturas entre L/8 y $\mathrm{L} / 5$ también es representativo, lo que habla de la tendencia de buscar puentes extradosados con características similares a puentes atirantados, ver figura 17. Sin embargo, a medida 


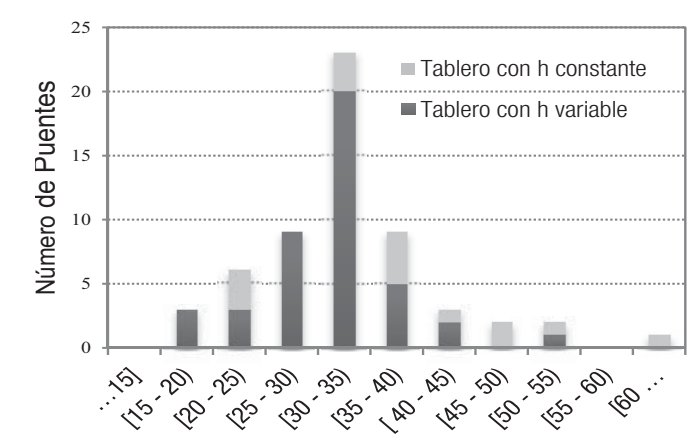

Esbeltez del tablero en la sección de apoyo (ha/L)

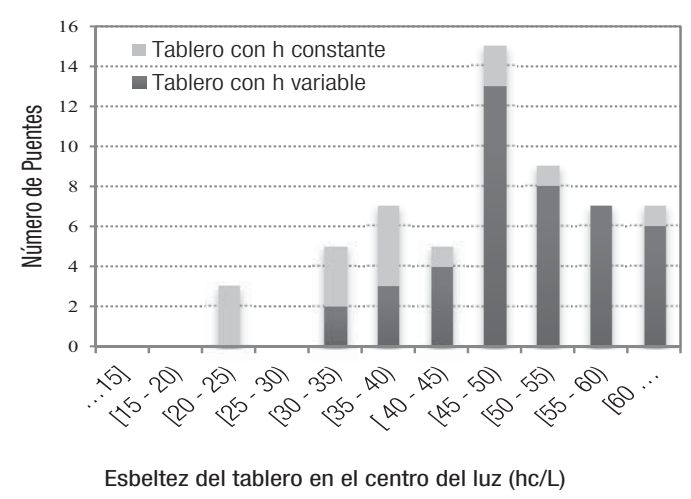

Esbeltez del tablero en el centro del luz (hc/L)

Figura 13. Relación de número de puentes según la esbeltez del tablero en la sección de apoyo en pila (izquierda) y en el vano central (derecha) para los puentes estudiados.

Fuente: elaboración propia

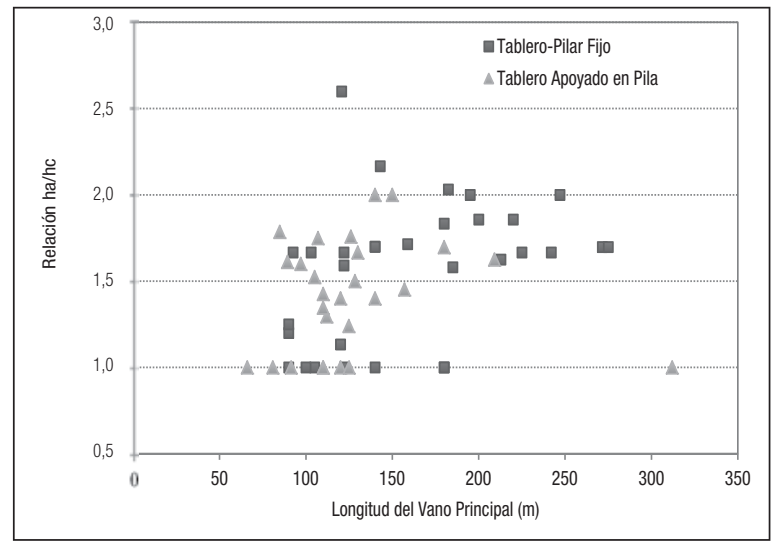

Figura 14. Relación ha/hc de acuerdo a la luz principal. Fuente: elaboración propia

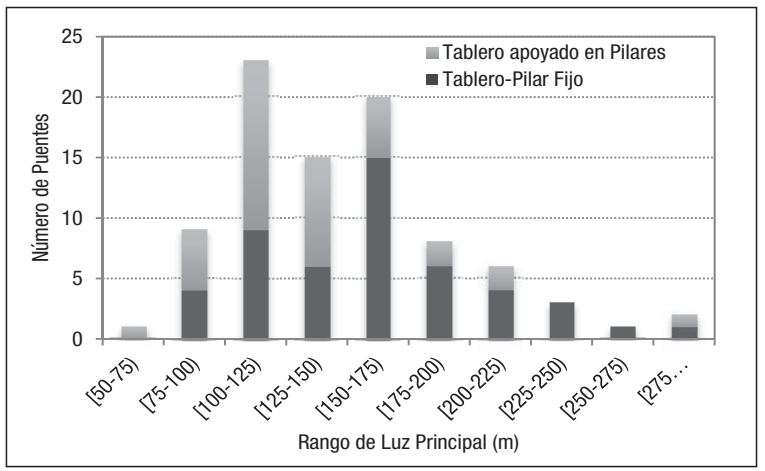

Figura 15. Relación de número de puentes de acuerdo al tipo de vinculación entre el tablero y el pilar.

Fuente: elaboración propia

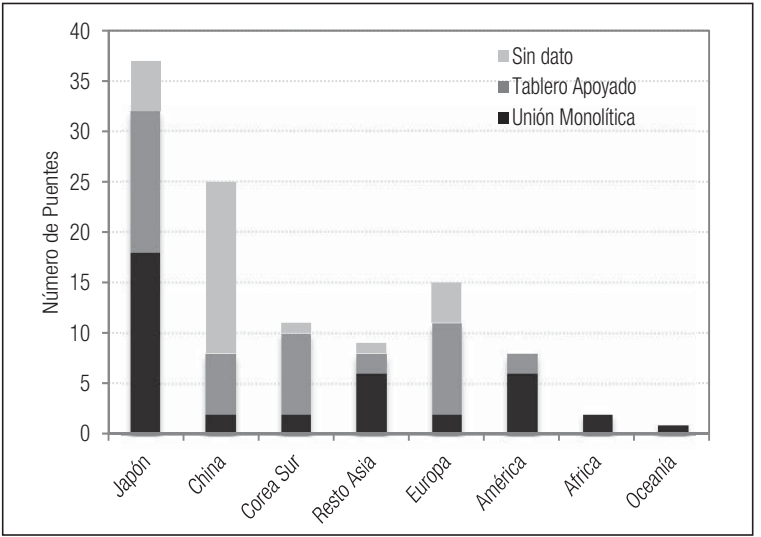

Figura 16. Relación del número de puentes construidos y en construcción en función del tipo de vinculación entre el tablero y los pilares, y de la región.

Fuente: elaboración propia

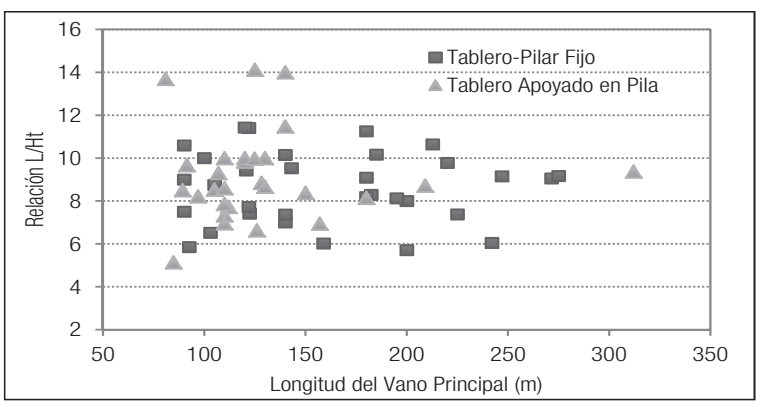

Figura 17. Relación luz principal/altura de la torre de acuerdo a la luz principal.

Fuente: elaboración propia 


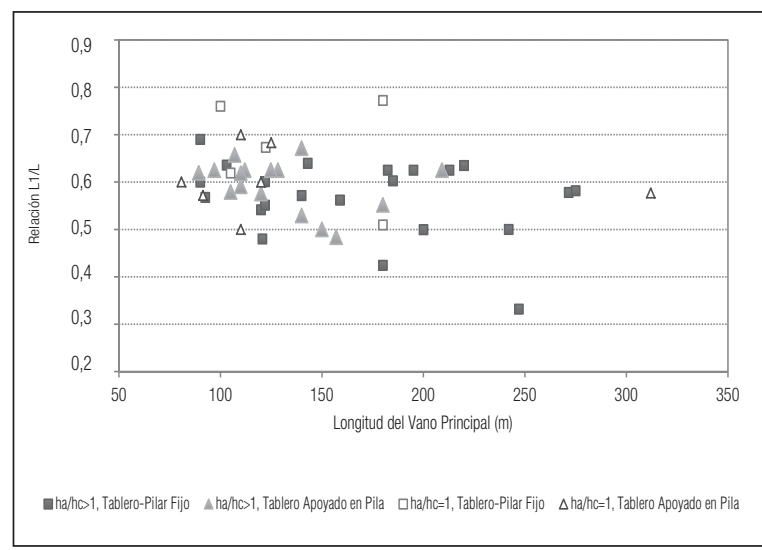

Figura 18. Relación longitud del vano lateral/longitud principal de acuerdo a la luz principal.

Fuente: elaboración propia

que se incrementa la luz del vano principal, la altura de la torre cae entre L/10 y L/8.

- a longitud del vano lateral no parece ser afectada por el tipo de vinculación entre el tablero y el pilar, ni por el grado de acartelamiento del tablero, ver figura 18. En general, se emplean vanos laterales de longitudes entre $0,5 \mathrm{~L}$ y $0,7 \mathrm{~L}$.

\section{VENTAJAS Y DESVENTAJAS}

Comparando los puentes extradosados con los atirantados y los pretensados de viga cajón, se pueden encontrar las siguientes ventajas:

- Una menor altura de la torre en los puentes extradosados, en comparación a los atirantados, ha permitido construir puentes en sitios cercanos a los aeropuertos. Además, al ser menor la altura de los pilonos se ofrece una reducción de la afectación visual del paisaje y una disminución en las labores de construcción.

- En comparación a los puentes pretensados de viga cajón, el extradosado es una solución más elegante, gracias a que la torre y los tirantes pueden crear una estructura emblemática.
- En el caso de suelos con baja capacidad portante, un puente extradosado resulta más favorable a un pretensado de viga cajón.

- Para puentes extradosados de tablero rígido construidos por voladizos sucesivos, gracias a la alta rigidez de la viga principal, al contrario de lo que ocurre en uno atirantado, no es necesario ajustar la fuerza de tensión de los cables atirantados, ni durante la obra, ni al finalizar la construcción del tablero.

- Debido a que morfológicamente un puente extradosado es una solución intermedia entre el atirantado y el de pretensado de viga cajón, mediante la modificación de la rigideces de los elementos estructurales principales, el ingeniero proyectista goza de cierta libertad para buscar el comportamiento deseado, el cual estará acotado por el comportamiento estructural de las otras dos tipologías.

- A su vez, las principales desventajas de los puentes extradosados se mencionan a continuación:

- Debido al mayor peso de la estructura, y a la no existencia de la estructura suspendida, como en los puentes atirantados, las fuerzas sísmicas sobre los elementos estructurales de los extradosados son mayores.

- Al no compensarse totalmente las cargas permanentes en un puente extradosado, se torna importante evaluar con sumo cuidado los fenómenos reológicos en el hormigón. En el caso de usar secciones híbridas, debido a las mayores fuerzas internas en un puente extradosado, en comparación de un atirantado del mismo vano principal, una gran parte de la fuerza axial en la viga, inicialmente resistida por la losa de concreto, fluye, a largo plazo, desde el hormigón hasta las vigas de acero [12]. Como resultado, se generan incrementos en la deflexión del vano principal, en la fuerza de los tirantes, y en la distribución de los esfuerzos longitudinales [57]. 


\section{CONCLUSIONES}

Los puentes extradosados han ganado un lugar como solución estructural para proyectos de carretera y ferrocarril, con luces entre 100 y 200 m, siendo competitivos con otras tipologías convencionales. En el continente asiático se ha dado gran acogida a esta tipología, donde Japón y China son los países con mayor número de realizaciones a nivel mundial. Recientes construcciones en Europa, América y África demuestran el despertar del interés por los puentes extradosados, sin embargo, en algunos países siguen siendo desconocidos.

Actualmente, en los puentes extradosados, construidos y en construcción, se refleja una preferencia por tableros de canto variable, de esbelteces entre L/30 y L/35 para la sección de apoyo, y entre L/45 y L/50 para la sección en el centro de luz. En cuanto al tipo de vinculación entre el tablero y el pilar, existe una fuerte paridad entre los dos tipos de vinculación típicos, a saber, conexión monolítica y tablero apoyado sobre los pilares, independientemente de la amenaza sísmica de la zona en que se construya el puente. Para el caso de tableros con canto constante, se prefieren esbelteces entre L/30 y L/40, y tableros apoyados sobre las pilas. Para la torre, aunque principalmente se emplean alturas entre $\mathrm{L} / 12 \mathrm{y} \mathrm{L} / 8$, el uso de alturas entre L/8 y L/5, permite entrever la búsqueda por un comportamiento similar al de los puentes atirantados, lo que demuestra la libertad en el diseño para un puente extradosado. Por último, se usan longitudes de vanos laterales entre el $50 \%$ y el $70 \%$ de la longitud del vano principal.

La evolución de estos puentes ha sido impulsada por el deseo de ampliar el rango de luz principal, aprovechando al máximo las bondades que ofrece la tipología. Para esto, surge como solución ideal, al igual que en los atirantados, la disminución del peso del tablero por medio de secciones metálicas o compuestas, o el uso de losas ortotrópicas en el centro del vano principal. Además, estos puentes han demostrado ser adaptables a procesos constructivos que difieren al de voladizos sucesivos. Sin embargo, a diferencia de los puentes atirantados, los fenómenos reológicos del hormigón toman una mayor importancia durante el diseño y construcción, lo cual obliga a estudios detallados y rigurosos para evitar problemas con la distribución de esfuerzos a largo plazo en los elementos.

\section{FINANCIAMIENTO}

Los autores agradecen a la Universidad Industrial de Santander (UIS), y al Departamento Administrativo de Ciencia, Tecnología e Innovación (COLCIENCIAS), por el apoyo económico recibido para la realización del proyecto de investigación titulado Aplicación de puentes de hormigón con pretensado extradosado en Colombia.

\section{REFERENCIAS}

[1] A. Ogawa, A. Kasuga, and H. Okamoto, "Prestressed Concrete Extradosed Bridge - Odawara Blueway Bridge", in XIII FIP Congress - National Report: Prestressed Concrete in Japan, Holland, 1998.

[2] J. Benjumea, G. Chio, y E. Maldonado, "Comportamiento Estructural y Criterios de Diseño de los Puentes Extradosados: Visión General y Estado del Arte," Revista Ingeniería de Construcción, Vol. 25, No 3, pp. 383-398, 2010.

[3] J. Llombart, y J. Revoltós, "Puente sobre el Río Deba," Revista de Obras Públicas, Vol. 152, pp. 71-74, 2005. 
[4] A. Grison, y J. Tonello, "A43: Pont de Saint-Rémy-de-Maurienne - Un parti original: la précontrainte extradossée," Travaux, Vol. 733, pp. 16-20, 1997.

[5] A. Kasuga, "Extradosed Bridges in Japan", Structural Concrete, Vol. 7, No 3, pp. 91103, 2006.

[6] D. Dos Santos, Comportamento Estrutural de Pontes com Pretensão no Extradorso, [Tesis de Mestrado], Escola Politécnica da Universidade de São Paulo, Brasil, 2006.

[7] J. Becze, y J. Barta, "Korong Prestressed Extradosed Bridge", Structural Engineering International, Vol. 16, No 1, pp. 28-30, 2006.

[8] J. Sobrino, "Puentes Atirantados y Extradosados," en III Simposio Internacional sobre Diseño y Construcción de Puentes, Universidad Industrial de Santander, Colombia, 2009.

[9] K. Yuyama, and J. Watanabe, "Innovative New Type of Cable Stayed Bridge - Yashiro Bridges of Hokuriku Shinkansen", in XIII FIP Congress - National Report: Prestressed Concrete in Japan, Holland, 1998.

[10] J. Binns, "Extradosed Bridge distinguishes Tollway Project in India," Civil Engineering Magazine, Vol. 75, No 2, p. 20, 2005.

[11] Kniga, et al., "Experience of Analysis of Bridge Structures", in $15^{\text {th }}$ International Conference on Computer Methods in Mechanics CMM-2003, Poland, 2003.

[12] K. Mermigas, Behaviour and Design of Extradosed Bridges, [MSc Thesis], University of Toronto, Canada, 2008.
[13] M. Nishimua, H. Mochizuki, Y. Iizuka and T. Akiyama, "Design and Construction of Sntanigawa Bridge -PC Extradosed-Type Bridge-", in $1^{s t}$ fib Congress: Concrete Structures for the $21^{\text {st }}$ Century, Japan, 2002.

[14] J. Lido, H. Nakayama, T. Wakasa, A. Kasuga, and K. Mizuno, "Design and Construction of Shin-Meisei Bridge", in the $1^{s t}$ fib Congress: Concrete Structures for the $21^{\text {st }}$ Century, Japan, 2002.

[15] P. Charlon, and J. Frappart, "Ravine des Trois Bassins-Bridge in La Reunion Island. A successful application of Extradosed Prestressing", in The International FIB Symposium, Holland, 2008.

[16] M. Tomita, K. Tei, and S. Takashi, "ShinKarato Bridge in Kobe", Structural Engineering International, Vol. 9, No 2, pp. 109-110, 1999.

[17] A. Ogawa, T. Matsuda and A. Kasuga, "The Tsukuhara Extradosed Bridge near Kobe", Structural Engineering International, Vol. 8, No 3, pp. 172-173, 1998.

[18] M. Kikuchi and T. Tabata, "Extradosed Prestressed Concrete Bridge - Kanizawa Bridge", in XIII FIP Congress - National Report: Prestressed Concrete in Japan, Holland, 1998.

[19] A. Griezic, C. Scollard, and D. Bergman, "Design of the Canada line Extradosed Transit Bridge", in $7^{\text {th }}$ International Conference on Short and Medium Span Bridges. Canada, 2006.

[20] M. Ishii, Sistemas Estruturais de Pontes Extradorso , [Tesis de Mestrado], Escola Politécnica da Universidade de São Paulo, Brasil, 2006. 
[21] N. Terada, T. Mochizuki, K. Komai and S. Nakamura, "The Design and Construction of the Miyakodagawa Bridge in the 2nd Tomei Expressway", in $1^{s t}$ fib Congress: Concrete Structures for the $21^{\text {st }}$ Century, Japan, 2002.

[22] OVM, "OVM Stay Cable System", [31 de Octubre de 2010], [Online], Available: http://fetech.net/Catalog\%20OVM/ OVM\%20STAY\%20CABLE\%20SYSTEM.pdf

[23] H. Oshima, N. Suzuki, T. Kashiwamura and I. Oda, "Design and Construction of the Japan-Palau Friendship Bridge", in $1^{s t}$ fib Congress: Concrete Structures for the $21{ }^{\text {st }}$ Century, Japan, 2002.

[24] S. Nakamura, Y. Momiyama, T. Hosaka and K. Homma, "New technologies of steel/concrete composite bridges" Journal of Constructional Steel Research, Vol. 58, No 1, pp. 99-130, 2002.

[25] Q. Fang, "Wuhu Double-Deck Cable Stayed Bridge", Structural Engineering International, Vol. 14, No 1, pp. 32-33, 2004.

[26] W. Kim, B. Joo, B. Kim and M. Choi, "Introduction of the First Extradosed Bridge in Korea", [En Coreano], Civil Engineering, Vol. 53, No 11, pp. 156-162, 2005.

[27] Haubans-Recommendations de la Commission Interministérielle de la Précontrainte, Service d'études Techniques des Routes et Autoroutes (SETRA), 2001.

[28] Acceptance of Stay Cable Systems using Prestressing Steels, International Federation for Structural Concrete (fib), 2005.

[29] Structurae, [1 de December de 2010], "Viaduc de la ravine des Trois-Bassins" in V.
Lesur [photographer] [Online]. Available: http://en.structurae.de/photos/index. cfm?JS=107351

[30] Connecticut Department of Transportation, [28 de March de 2010], "I-95 New Haven Harbor Crossing Corridor Improvement Program", [Online]. Available: http://www. i95newhaven.com/pdfs/features_home/ qbridgenews_1.pdf

[31] Minnesota Department of Transportation, [20 de March de 2010], "I-95 St. Croix River Crossing Project: Updated Schedule", [Online]. Available : http://www.dot.state. mn.us/metro/projects/stcroix/pdfs/schedule2-18-10.pdf.

[32] ITN Source, [22 de December de 2009], "Ethiopia: Inauguration of new 303-metre Abay Bridge acros Blue Nile linking Sudan to Ethiopia, easing transport and boosting trade between two countries", [Online]. Available: http://www. itnsource.com/shotlist/RTV/2008/09/12/ RTV3074908/?v=0\&a=1.

[33] JICA, Japan International Cooperation Agency, [12 de February de 2010], "Region-Specific Activities and Initiavitves: The Middle East"; [Online]. Available: http://www.jica.go.jp/english/publications/ reports/annual/2009/pdf/15.pdf

[34] PEDELTA, [15 de Marzo de 2011], "Puente Los Trillizos, La Paz, Bolivia", [Online]. Available: http://www.pedelta.es/es/Proyectos/Puentes/Carretera/trillizos

[35] Structurae, [28 de Agosto de 2010], "Deba River Bridge", in J. Llombart, [ photographer], [Online]. Available: http://en.structurae.de/photos/index. cfm?JS=50811 
[36] J. Strasky, "The power of Prestressing", Structural Concrete, Vol. 4, No 1, pp. 2543, 2003.

[37] Structurae, [14 March de 2011], "Sunniberg Bridge", in A. Ruiz, [photographer], [Online]. Available: http://en.structurae.de/ photos/index.cfm?JS=130322

[38] A. Kasuga, "Construction of Extradosed Bridges in Japan", in Seminar on Design and Construction of Prestressed Concrete Cable-Stayed Bridges, Malayisia, 2002.

[39] Easytourchina, "Wuhu Changjiang Bridge", [5 de May de 2011], [Online]. Available: http://www.flickr.com/photos/44847207@N00/3284334632/in/ photostream

[40] T. Yoshioka, "The latest Technologies of Prestressed Concrete Bridges in Japan", in JSCE-VIFCEA Joint Seminar on Concrete Engineering, Vietnam, 2005.

[41] J. Combault, et al., "Pont sur la Charente à Cognac",Travaux, Vol.636,pp. 31-37, 1988.

[42] H. Mutsuyoshi, and H. Minh, "Prestressed Concrete Bridges in Japan: Technological Advancements and Future Prospects", in $3^{\text {rd }} A C F$ International Conference- $A C F /$ $V C A$, Vietnam, 2008.

[43] Y. Maeda, Y. Imaizumi, A. Kasuga and K. Tazoe, "Design and Construction of the Himi Bridge -Extradosed Bridge with Corrugated Steel Web-", in $1^{s t}$ fib Congress: Concrete Structures for the $21^{\text {st }}$ Century, Japan, 2002.

[44] Y. Maeda, M. Iijima, A. Hiroshi and A. Kasuga, "The Extradosed Cable Anchorage and Ultimate Behavior for Extradosed Bridge with Corrugated Steel Webs", [En
Japonés], Proceedings of JSCE, Vol. 794, pp. 227-238, 2005.

[45] Tabikappa.blog, [5 de March de 2010], "Himi Yume Bridge". [Online]. Available: http://tabikappa.blog55.fc2.com/blog-entry-744.html

[46] K. Trimbath, "Bridges: Hybrid Extradosed Bridge under way in British Columbia", Civil Engineering Magazine, Vol. 76, No. 10, pp. 28-30, 2006.

[47] Structurae, [22 de Diciembre de 2010], "Golden Ears Bridge, Vancouver"; in D. Feldman [photographer], [Online]. Available: http://en.structurae.de/photos/index. cfm?JS=155421

[48] A. Tsukamoto, H. Okagawa, S. Yoshino and Y. Konishi, "Construction of an Extradosed Bridge by Incremental Launching Method", in $1^{\text {st }}$ fib Congress: Concrete Structures for the $21^{\text {st }}$ Century, Japan, 2002.

[49] A. Gridnev, [22 de February 2010], "The South Bridge Project, Riga, Latvia", [Online]. Available: http://www.balticroads.org/ conference27/papers/27BRC_B2_Gridnevs.pdf

[50] M. Virlogeux, "Recent evolution of cablestayed bridges," Engineering Structures, vol. 21, no 8, pp. 737-755, 1999.

[51] H. Otsuka, T. Wakasa, J. Ogata, W. Yabuki, and D. Takemura, "Comparison of structural characteristics for different types of cable-supported prestressed concrete bridges", Structural Concrete, Vol. 3, No. 1, pp. 3-21, 2002.

[52] K. Umezu, I. Sakai, M. Fujita, H. Arai and J. Yamazaki, "Study on Structural Char- 
acteristic of Prestressed Concrete Girder Bridges with External Tendons of Large Eccentricity", [En Japonés], Proceedings of JSCE, Vol. 788, pp. 139-158, 2005.

[53] U. Meiss, Anwendung von Strukturoptimierungsmethoden auf den Entwurf mehrfeldriger Schrägseilbrücken und Extradosed Bridges, [Doctoral Dissertation], University of Stuttgart, Germany, 2007.

[54] J. Mathivat, "Recent Developments in Prestressed Concrete Bridges", FIP Notes, Vol. 2, pp. 15-21, 1988.

[55] G. Chio, Comportamiento Estructural y Criterios de Diseño de los Puentes con
Pretensado Extradosado, [Tesis Doctoral], Universidad Politécnica de Cataluña, España, 2000.

[56] M. Komiya, “エクストラドーズド PC 道路橋の設計に関する一考察” (Study for the Design of Extradosed PC Highway Bridges), [En Japonés], Journal of Construction Management and Engineering, Vol. 516, No. VI-27, pp. 27-39, 1995.

[57] K. Takami and S. Hamada, "Long-Time Behavior of Extradosed Composite Birdges", in Cable-Supported Bridges - Challenging Technical Limits: IABSE Conference, South Korea, 2001. 